\title{
Infiltration of CD68+ cells correlates positively with matrix metalloproteinase 2 expression in the arteries used as aortocoronary bypass grafts. Possible clinical implications
}

Bartłomiej Perek ${ }^{1}$, Katarzyna Kowalska ${ }^{2}$, Bartosz Kempisty ${ }^{2,3}$, Mariusz Nawrocki ${ }^{3}$, Michał Nowicki ${ }^{2}$, Mateusz Puślecki ${ }^{1,4}$, Danuta Ostalska-Nowicka ${ }^{5}$, Łukasz Szarpak ${ }^{6}$, Navid Ahmadi ${ }^{1}$, Agnieszka Malińska ${ }^{2}$

${ }^{1}$ Department of Cardiac Surgery and Transplantology, Poznan University of Medical Sciences, Poznan, Poland

${ }^{2}$ Department of Histology and Embryology, Poznan University of Medical Sciences, Poznan, Poland

${ }^{3}$ Department of Anatomy, Poznan University of Medical Sciences, Poznan, Poland

${ }^{4}$ Department of Medical Rescue, Poznan University of Medical Sciences, Poznan, Poland

${ }^{5}$ Department of Pediatric Nephrology, Poznan University of Medical Sciences, Poznan, Poland

${ }^{6}$ Lazarski University, Warsaw, Poland

\begin{abstract}
Background: Late failure of arterial aortocoronary conduits may result from abnormal activity of cells found in the vessel wall, including macrophages. The purpose of this study was to assess if there are any associations between the number of macrophages and overexpression of matrix metalloproteinases (MMPs) in the wall of arterial grafts, as well as their clinical significance.

Methods: This study involved 128 consecutive patients with a mean age of $64.9 \pm 9.7$ years who underwent elective surgery for coronary artery disease (CAD). The surplus segments of internal thoracic artery (ITA) and radial arteries (RA) were taken for immunohistochemical analysis of macrophage numbers and MMPs expression. The participants who reached the clinical primary end-point (cardiacrelated death, acute coronary syndrome or progression of CAD) had a follow-up angiography.

Results: The mean numbers of macrophages were higher on RA (70 [24; 112]) than ITA cross-sections (44 [24; 59]; $p<0.001)$. Median expression of both MMP2 and MMP9 were stronger in the ITA than $R A$ cross-sections $(p<0.001)$. A significant positive correlation of MMP2 expression and a number of macrophages infiltrating the tunica media of arterial segments were noted on both ITA and RA cross-sections. In addition, the arterial segments of the 6 patients who reached clinical end-point had higher numbers of macrophages and stronger MMP2 expression when compared to the rest of the participants.

Conclusions: Macrophage infiltration of arterial wall grafts prior to harvesting may be associated with higher risk of late occlusion and MMP2 might be facilitating this process. (Cardiol J 2020; 27, 6: 817-824)
\end{abstract}

Key words: coronary artery bypass grafting, arterial aortocoronary grafts, macrophage, matrix metalloproteinase, outcomes

Address for correspondence: Bartłomiej Perek, MD, PhD, Department of Cardiac Surgery and Transplantology, Poznan University of Medical Sciences, ul. Długa 1/2, 61-848 Poznań, Poland, tel: +48 6185492 10, e-mail: bperek@ump.edu.pl Received: 3.12 .2018

Accepted: 27.03.2019 


\section{Introduction}

Coronary artery bypass grafting (CABG) is a method of choice in treating patients with severe coronary artery disease (CAD) [1]. Late adverse outcomes of CABG are determined by durability of aortocoronary conduits as well as progression of atherosclerosis in the native coronary arteries [2, 3]. Due to excellent long-term patency in follow up, the left internal thoracic artery (LITA) implanted in the left anterior descending artery (LAD) has been the gold standard in cardiac surgery for many years [4]. The angiographic and clinical results of the other arteries or veins as a second graft in CABG patients shows a lower efficacy than internal thoracic artery (ITA) [5, 6]. According to the findings of ultrastructural studies, ITA differs from other vessels, not only in morphological terms but also in physiological features, these features make it an exceptional vessel with intrinsic resistance to atherosclerotic degeneration [7].

One of the many theories suggests that, an imbalance in local hemostasis between matrix metalloproteinases (MMPs) and locally released inhibitors, so called tissue inhibitor of metalloproteinases (TIMPs) leads to many pathologies in the vessel wall. In particular, MMP2 and MMP9 known as gelatinase-A and gelatinase-B, respectively, were shown to play an important role in the rupture of atherosclerotic plaques (leading to stroke or acute coronary syndrome), acute aortic dissection and leg venous ulcers [8-11]. Moreover, reduced production of macrophage-derived gelatinases was found to be associated with a significant decrease in plaque area and inhibition of cerebral aneurysm formation in animal experimental models [12, 13]. Although MMPs are expressed in almost all tissues of the human body, their synthesis takes place predominantly in macrophages, endothelial cells and smooth muscle cells [14].

Recent studies have demonstrated that infiltration of the coronary arteries' walls by macrophages is one of the fundamental step in the development of atherosclerosis [15]. Additionally, the presence of CD68 + cells (not yet developed into foam cells) in the intima of the grafted saphenous veins were shown to serve as one of the earliest markers for detection of graft occlusion [16].

In light of the above discussion, it was decided to evaluate the association between macrophage infiltration and overexpression of MMPs in the walls of arteries applied routinely as aortocoronary conduits. The aim will be to eventually determine any potential clinical significance of this phenomenon.
Table 1. Basic preoperative data of examined patients.

\begin{tabular}{lc}
\hline Variables & N = 128 \\
\hline Age [years] & $64.9 \pm 9.7$ \\
Obesity (BMI > 30) & $52(40.6 \%)$ \\
Arterial hypertension & $83(64.8 \%)$ \\
Diabetes mellitus treated with insulin & $33(25.8 \%)$ \\
Hyperlipidemia & $54(42.2 \%)$ \\
Neurological events* & $11(8.6 \%)$ \\
Peripheral vascular disease & $30(23.4 \%)$ \\
Chronic obstructive disease & $17(13.3 \%)$ \\
Renal failure ${ }^{*}$ & $14(10.9 \%)$ \\
Active smoking*** & $63(49.2 \%)$ \\
\hline
\end{tabular}

Continuous variables are presented as mean \pm standard deviation, whereas categorical values are presented as the numbers (percentages). * They refer to both strokes and transient ischemic attacks; **When estimated glomerular filtration rate was below $60 \mathrm{~mL} /$ $/ \mathrm{min} / 1.73 \mathrm{~m}^{2}$; ***The term "active smokers" comprises active smokers and individuals who had given up smoking within 1 year prior to surgery; BMI — body mass index

\section{Methods}

This study involved 128 consecutive patients (100 men and 28 women) with a mean age of $64.9 \pm$ \pm 9.7 years (ranged 42 through 86 ) who underwent elective isolated $\mathrm{CABG}$ procedures in one cardiac surgical center in the years 2009-2011. Patients were qualified for surgery on the basis of coronary angiography. Basic preoperative data are presented in Table 1.

The study protocol was approved by the Local Bioethical Committee (No. 1201/08) and each patient provided informed written consent for participation in the study.

\section{Biological material obtaining and preparation}

During CABG procedures, surplus segments of vessels used as aortocoronary grafts were taken for ultrastructural studies. In all cases, the most distal excess segments of at least one centimeter in length were harvested. Both arteries were dissected free as pedicled grafts. Radial arteries (RA) were obtained from a full skin incision over its entire course. To minimize possible damage to the vessels, surgeons avoided: touching (no-touch technique), excessive manipulation, dilatation, and using high-energy electrocautery. Ultimately 174 segments of vessels, including 128 ITA and 46 RA were saved for histological analysis.

Segments of obtained vessels that passed intraoperative macroscopic inspection for any ab- 
normalities were carefully rinsed with $0.9 \% \mathrm{NaCl}$ at room temperature, slightly dilated, then immersed and fixed in freshly prepared Bouin's solution. The remaining preparatory steps for light microscope examination were described in detail in a previous paper [16]. All immunohistochemical analyses utilized the Dako REAL EnVision Detection System, Peroxidase/DAB, Rabbit/Mouse, K5007 (Dako, Copenhagen, Denmark) and were prepared according to standard procedure [16]. The following mouse monoclonal antibodies: anti-MMP2 (dilution 1:50; NB200-114), anti-MMP9 (dilution 1:250; NB10078556, both Novus Biologicals, Littleton, United States), and anti-CD68 antibody (dilution 1:100, M0814, Dako) were used. Additionally, an eliminating assay with the following specific antibodies for lymphocyte subpopulations such as anti-CD20cy (1:400 dilution, M0755, Dako), anti-CD3 (1:50 dilution, M7254, Dako), anti-CD8 (1:100 dilution, M7103, Dako) and anti-CD30 (1:40 dilution, M0751, Dako) was carried out [17]. The peroxidase reaction was developed using diaminobenzidine.

\section{Immunohistochemical analysis}

Transverse sections of the arteries were observed and analyzed under a brightfield microscope-Olympus BX 50 (OLYMPUS Optical Europe, Germany) equipped with a Mirax-Midi scanner (Carl Zeiss Microimaging GmbH, Germany), coupled with a Panoramic Viewer, version 1.15.4, software (3DHISTECH Ltd., Budapest, Hungary).

The expression of CD68 + cells (a macrophage specific protein) were analyzed throughout the grafted vessel wall, followed by the inspection of specific sections such as; the tunica intima, the tunica media and the tunica adventitia. The intensity of cytoplasmic expression of the MMPs was assessed by applying the semi-quantitative immunoreactive score (IRS) scale according to Remmele and Stegner [18]. This method takes into account the percentage of positive cells (scale from 0 to 4 ), and intensity of the color reaction (scale from 0 to 3 ), to produce a final score ranging from 0 to 12 , encapsulating points given for individual traits. According to IRS, the expression of the cytoplasmic proteins is classified as negative (IRS 0-1), weak (IRS 2-3), moderate (IRS 4-6) or strong (IRS 8-12).

For every segment analyzed, at least 8 to 10 representative sections were considered in making data more reliable. Immunohistochemical evaluation of protein expression was carried out by two experienced histologists, through blind sample analysis, based on encoded numbers corresponding with the basic rules of positive and negative control. The negative controls were carried out in an identical way to the experimental sample, with the exclusion of primary antibody being replaced with normal mouse serum.

\section{Postoperative outcomes}

All patients after discharge were systematically treated in the outpatient clinic. Special attention was paid to control CAD symptoms. If participants reached the clinical study primary endpoint, defined as cardiac-related death, acute coronary syndrome or necessity to carry out coronary angiography due to progression of angina according to Canadian Cardiovascular Society (CCS) scale, a follow-up angiography was performed.

\section{Data management and statistical analysis}

The Shapiro-Wilk W test for normality was performed for all continuous variables. These normally distributed data were presented as a mean and standard deviation. Data that did not satisfy the criteria of normal distribution was expressed as a median with the $25^{\text {th }}$ and $75^{\text {th }}$ percentiles, which was compared with the Mann-Whitney U test. Similarly, categorical variables (IRS scale) were presented as medians with the $25^{\text {th }}$ and $75^{\text {th }}$ percentiles. Both types of variables were compared with the non-parametric Mann-Whitney U test. Dychotomic data are presented as numbers (n) and percentages $(\%)$. The correlation between the findings of MMPs expression and CD68+ cells on the arterial sections were tested using the Spearman $\mathrm{R}$ correlation method. The correlation was defined as 'very strong' when $\mathrm{R}$ was between 0.8 and 1.0 (or -0.8 and -1.0 ), 'strong' between 0.6 and 0.8 , or moderate between 0.4 and 0.6 .

A p value below 0.05 was considered statistically significant. These statistical analyses were carried out using the Statistica 10.0 Package for Windows (StatSoft, Inc., Tulsa, OK, USA).

\section{Results}

\section{CD68+ cells}

The mean number of $\mathrm{CD} 68+$ cells were higher on RA $(70[24 ; 112])$ than ITA $(44[24 ; 59])$ crosssections $(p<0.001)$. A detailed analysis to visualize the presence of these cells in both arteries showed a higher number of CD68 + cells in both the tunica media and tunica adventitia, while comparable in the tunica intima (Table 2). In both types of arteries, approximately $70 \%$ of $\mathrm{CD} 68+$ cells were found in the tunica adventitia (Fig. 1); 
Table 2. CD68+ cell counts and matrix metalloproteinases (MMPs) expressions on arterial cross-sections.

\begin{tabular}{lcccc}
\hline CD68+ cells* & Tunica intima & Tunica media & Tunica adventitia & TN or ME* $^{*}$ \\
\hline ITA $(n=128)$ & $5(3,12)$ & $4(2,10)$ & $28(17,44)$ & $44(24,59)$ \\
RA $(n=46)$ & $4(2,13)$ & $12(5,16)$ & $46(20,84)$ & $70(24,112)$ \\
P** $^{*}=$ & $<0.001$ & $<0.001$ & $<0.001$ \\
MMP2*** & 0.739 & & $1(0,2)$ & $1(0,2)$ \\
ITA $(n=128)$ & $1(0,2)$ & $4(0,2)$ & $2(1,2)$ & $4(2,4)$ \\
RA $(n=46)$ & $4(2,4)$ & $<0.001$ & $<0.001$ & $<0.001$ \\
P & $<0.001$ & & & $2(1,4)$ \\
MMP9 & & $2(2,4)$ & $1(1,2)$ & $3(2,4)$ \\
ITA $(n=128)$ & $2(1,2)$ & $6(4,6)$ & $2(1,2)$ & 0.004 \\
RA $(n=46)$ & $3(2,4)$ & $<0.001$ & 0.010 & \\
P & $<0.001$ & & & \\
\hline
\end{tabular}

${ }^{*}$ Cell counts are expressed as median $\left(25^{\text {th }}\right.$ percentile, $75^{\text {th }}$ percentile $) ; *$ ITA vs. RA; ***MMPs expressions are expressed as median $\left(25^{\text {th }}\right.$ percentile, $75^{\text {th }}$ percentile) of IRS points; \#Total number (TN) refers to CD68+ cells whereas median expression (ME) to MMPs; ITA — internal thoracic artery; RA - radial artery

Table 3. Correlations between matrix metalloproteinase 2 (MMP2) expression and CD68+ cells on cross-sections of arterial grafts.

\begin{tabular}{lcccc}
\hline Number of CD68+ cells & Tunica intima & Tunica media & Tunica adventitia & Total number \\
\hline MMP2 (ITA) & NS* & $r=0.409 ; p=0.034$ & NS & $r=0.426 ; p=0.029$ \\
MMP2 (RA) & NS & $r=0.429 ; p=0.001$ & NS & $r=0.467 ; p=0.006$ \\
\hline
\end{tabular}

*NS when $r$ index was below 0.400 and $p$ value did not exceed 0.05 ; ITA — internal thoracic artery; RA — radial artery; NS — non-significant

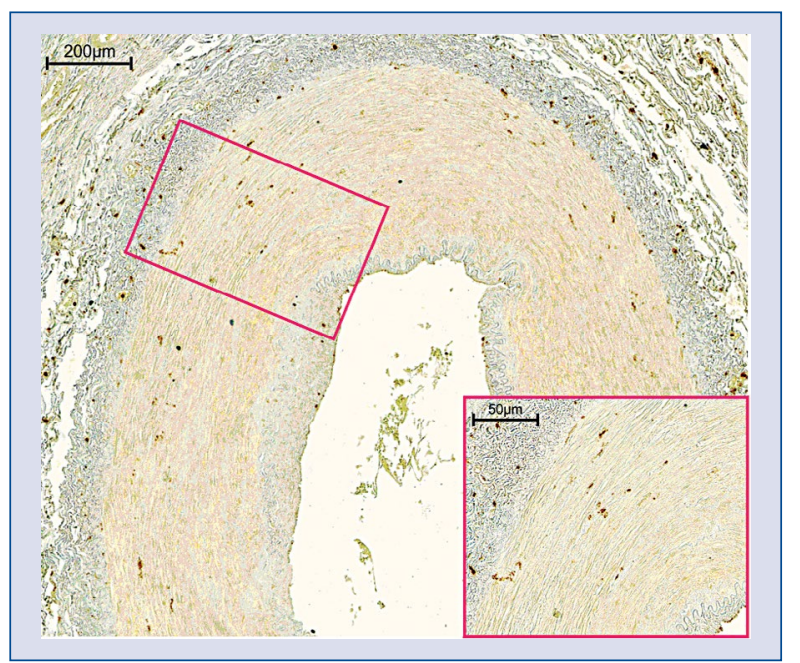

Figure 1. CD68+ cells on the radial artery cross-section. A radial artery segment was harvested from a 55-year-old man with triple-vessel disease. CD68+ cells were found predominantly in the tunica adventitia and outer layers of the tunica media.

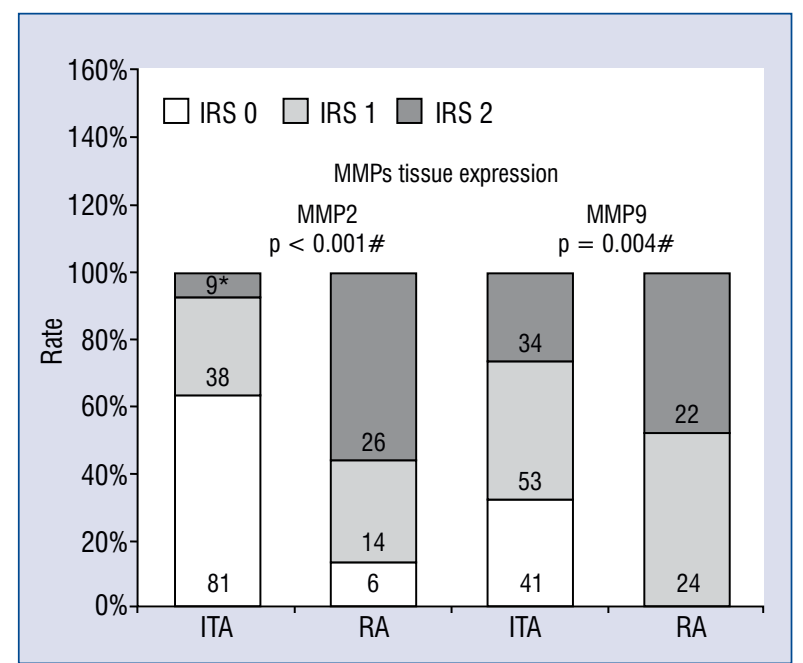

Figure 2. A rate of arterial cross-sections with different immunoreactive score (IRS) of matrix metalloproteinase (MMPs) expressions. More cross-sections of radial artery (RA) as compared to internal thoracic artery (ITA) presented higher IRS for both MMP2 and MMP9 immunostaining; *a number of cross-sections with a given IRS; \#ITA vs. RA. 


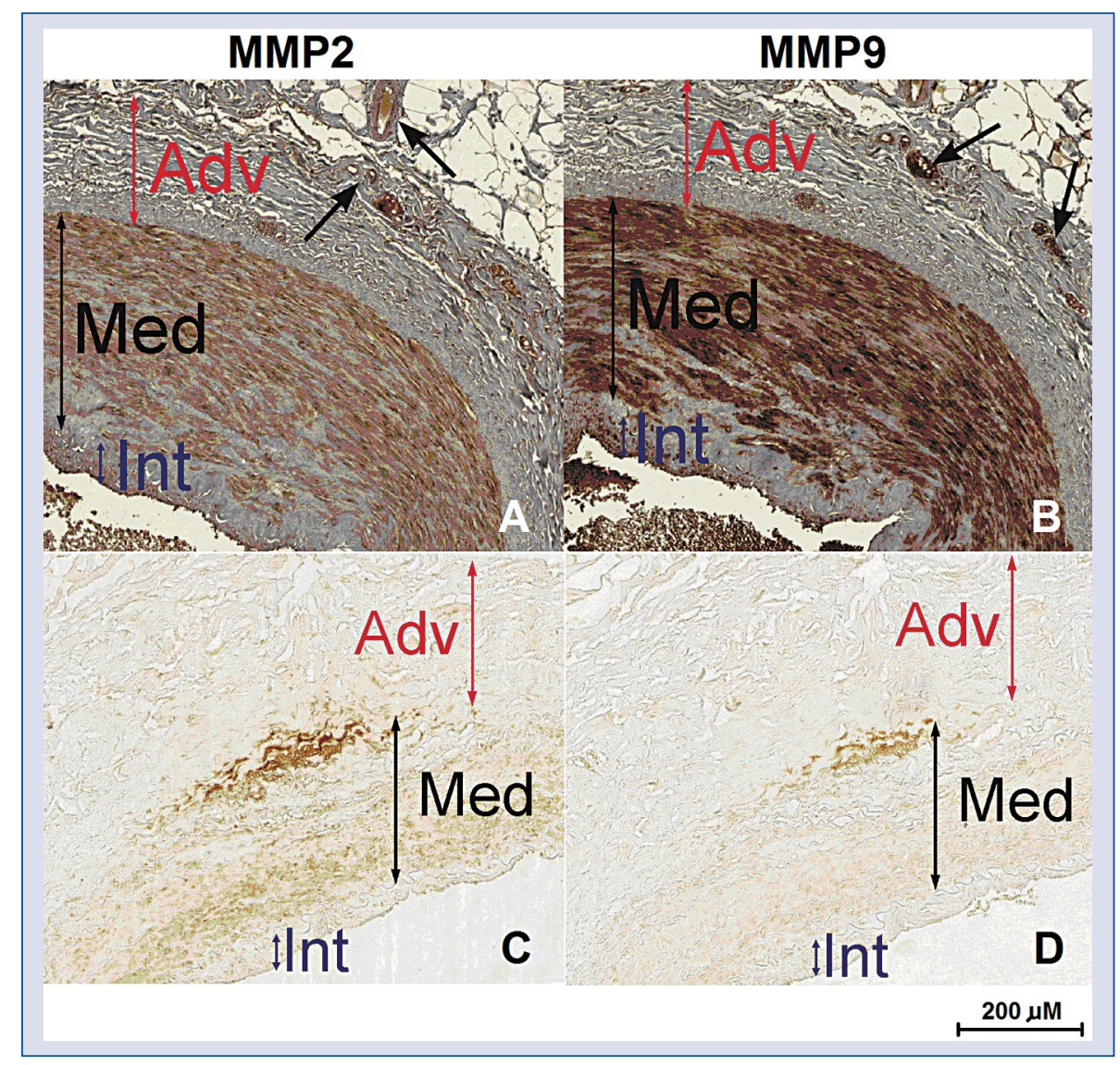

Figure 3. Immunoexpression of matrix metalloproteinase (MMPs) on the arterial cross-sections. The surplus arterial segments were obtained from 72-year-old man with two-vessel disease (severe stenosis in the left anterior descending artery and chronic totally occluded right coronary artery). Tissue expressions of MMP2 (A) and MMP9 (B) in the tunica media (Med) of radial artery (RA) cross-sections were found to be much stronger than in internal thoracic artery wall; C. MMP2; D. MMP9. On the RA sections, MMPs-positive cells were also seen in the wall of vasa vasorum (arrows); Adv — tunica adventitia; Int — tunica intima.

of note, these cells were mainly found adjacent to the vasa vasorum.

\section{Gelatinases expression}

Median expressions of MMP2 using Remmele scale (IRS) for ITA and RA cross-sections were minimal $(1[0,2])$ and moderate $(4[2,4])$, respectively $(\mathrm{p}<0.001)$. The same differences were seen in all layers of the arteries (Table 3 ). No expression of MMP2 (IRS 0-1 points) was noticed in the majority of ITA $(81 / 128)$ while in less than $15 \%$ of RA (6/26) segments (Fig. 2). Similar marked differences were detected for tissue expression of MMP9 (Table 2, Figs. 2 and 3).

\section{Correlation between number of CD68+ cells and gelatinases expression}

A significant positive correlation was noted between the total number of CD68+ cells and MMP2 expression in both ITA and RA cross-sections, as well as its presence in the tunica media of both arteries (Table 3; Fig. 4). No such correlation was observed between macrophage infiltration and immunostaining for MMP9.

\section{Long-term outcomes}

\section{and histological findings}

Based on the findings of adverse late outcomes of CABG, 25 out of 128 patients reached the primary clinical end-point. From these, coronary angiography of 6 patients revealed an underlying progression of CAD of the native arteries (not addressed during primary surgery), was responsible for their clinical deterioration. The rest of the remaining patients $(n=19)$, significant occluding lesions were visualized; predominantly in venous grafts $(n=17)$ followed by RA $(n=4)$ and in only two cases of ITA segments. 


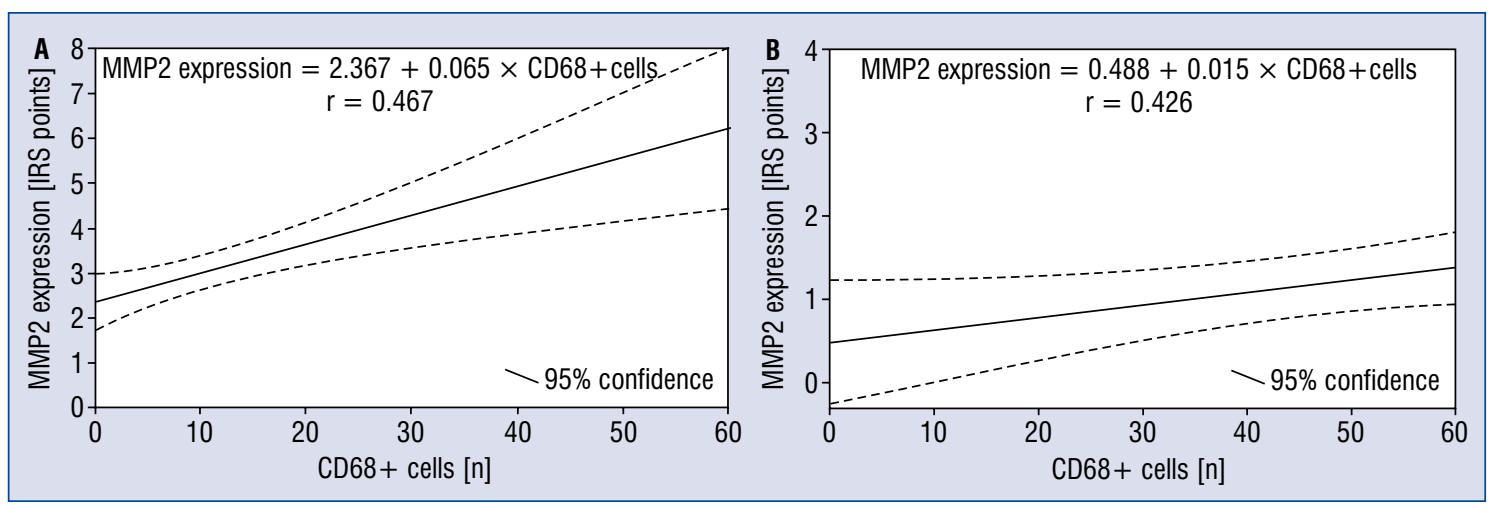

Figure 4. Correlation between matrix metalloproteinase 2 (MMP2) expression and number of CD68+ cells. Moderate correlations were found between median of MMP2 expression and a total number of CD68+ cells. Of note, MMP2 tissue expression was usually higher on the radial artery $(\mathbf{A})$ than on the internal thoracic artery $(B)$ sections.

Table 4. Number of CD68+ cells and matrix metalloproteinase (MMPs) expression in patients with occluded aortocoronary grafts.

\begin{tabular}{lcccccc}
\hline & $\begin{array}{c}\text { CD68 }+ \\
\text { Total [n] }\end{array}$ & $\begin{array}{c}\text { CD68+ } \\
\text { Int [n] }\end{array}$ & $\begin{array}{c}\text { CD68+ } \\
\text { Med [n] }\end{array}$ & $\begin{array}{c}\text { CD68+ } \\
\text { Adv [n] }\end{array}$ & $\begin{array}{c}\text { MMP2 } \\
{[\text { IRS ] }}\end{array}$ & $\begin{array}{c}\text { MMP9 } \\
{[\text { IRS ] }}\end{array}$ \\
\hline ITA 1 & 49 & 11 & 14 & 24 & 4 & 3 \\
ITA 2 & 62 & 12 & 20 & 30 & 6 & 2 \\
RA 1 & 74 & 10 & 27 & 37 & 4 & 4 \\
RA 2 & 85 & 14 & 22 & 49 & 6 & 6 \\
RA 3 & 72 & 12 & 19 & 41 & 6 & 4 \\
RA 4 & 115 & 22 & 31 & 51 & 6 & 4 \\
\hline
\end{tabular}

Adv — tunica adventitia; Int — tunica intima; IRS — immunoreactive score; ITA — internal thoracic artery; Med — tunica media; RA — radial artery

Due to a small number of arterial conduits with significant lesions, no reliable statistical analysis was possible. However, all detailed results of histological assessment and calculations are summarized in Table 4. In all cases reaching the study clinical end-point, arterial segments before their implantation had more CD68 + cells infiltrating the wall accompanied by higher MMPs expressions than median values for a whole group. Additionally, CD68 + cells were localized closer to inner layers of the vessels since their rate in tunica adventitia $(44.3 \%$ to $56.7 \%)$ was lower than in the rest of arterial cross-sections (approximately 70\%).

\section{Discussion}

The first finding of the present study showed that the total number of CD68+ cells was higher in RA than ITA walls. It is highly likely that these cells were macrophages since an eliminating assay was applied. Previous research has found that macrophages participate actively in early stages of vessel wall degeneration, including trans-differentiation, proliferation, microcalcification and migration of vascular smooth muscle cells $[18,19]$. Consequently, it is thought that RA conduits composed of a larger percentage of macrophages in the wall prior to their implantation into the coronary arteries are more prone to atherosclerotic degeneration and eventually graft failure. It has been shown in the past, arterial grafts have better long-term prognosis than saphenous vein grafts used for CABG [3, 4]. However, the current study confirmed that even arterial aortocoronary conduits, with pre-existing infiltration by CD68+ should be considered a risk factor of failure in a late follow-up period. It should be stressed again, all conduits which were closed or severely stenotic in the follow-up examination had a higher mean number of macrophages than the mean number in the whole group of examined conduits. However, due to a relatively small number of failed grafts, reliable statistical analysis was not possible. Impressively, the rate of medial and intimal macrophages in these grafts did increase 
from about $30 \%$ to approximately $50 \%$. Currently, this study is unable to explain if this phenomenon has any significance, nonetheless it was convincing that this finding warrants further research by applying more sophisticated scientific tools such as transmission electron microscopy.

Macrophages may infiltrate the vessel wall from the lumen or from the adventitial vasa vasorum. Current evidence suggests that the latter microvessels do not penetrate arterial walls, thus making it a preferred vessel compared to veins [20]. Concurrently, contrary to RA, internal elastic lamina is a tight structure in ITA, physiologically making it non-permeable [21]. This fact could be a possible explanation for differences seen in macrophage representation in the tunica media of both arteries. The harvested ITA conduits, which failed within post-discharge period could be due to pre-existing negative microstructural changes such as disruption of internal elastic lamina, which allowed a high number of CD68+ cells to penetrate through this layer. Once again, a more detailed, higher resolution histological study should be carried out to confirm such a brave hypothesis.

The next finding of possible clinical significance was a marked higher expression of MMP2 and MMP9 in the RA than ITA segments. Both MMPs produced by macrophages, with unique abilities to degrade elastin and collagen (the main components of extracellular matrix), are of paramount importance in many pathological processes, including atherosclerosis development and negative remodeling of venous graft applied extensively in CABG procedures [22]. Turner et al. [23] showed selective gene silencing of either MMP2 or MMP9 markedly reduced the invasive capacity of cultured human saphenous vein-smooth muscle cells (SMC), indicating that these MMPs played distinct non-overlapping roles in venous SMC invasion in vitro. Although on a very limited number of vessels, it was shown that some patients developed significant stenosis in graft within the follow-up period. This might be due to abnormally increased tissue activity of MMPs, which may also impact the outcome of arterial aortocoronary grafts. Stronger immunoreactivity for MMPs on the RA than ITA cross-sections should be considered as the next scientific proof that the latter is less prone to negative remodeling, leading to graft failure.

A positive correlation between a number of CD68+ cells in the whole wall (also in the tunica media) and tissue MMP2 expression was found. However, comparing histological views of CD68+ cells distribution and MMPs immunoreactivity on the vessel cross-sections, it is not possible that MMPs-positive areas were covered exclusively by macrophages. It can be suggested that CD68+ cells did stimulate SMCs to produce and release MMPs.

\section{Limitations of the study}

There are limitations in this study. Immunohistological assessment of the protein expression was carried out before implantation into aortocoronary circulation. Ideally, such analysis would be performed on the grafts explanted after a given period of time. It is possible to carry out such studies, but only in experimental animal models. Tissue expression was assessed on a base of immunohistochemistry. Although this method is accepted as a scientific tool to evaluate protein expressions, it is qualitative rather than quantitative one. While the Remmele scale applied improves quality of the analysis, experience of research remains mandatory. Histologists involved in this project may legitimate their expertise with many previous peer-reviewed publications [22, 24].

\section{Conclusions}

Infiltration of the walls of arterial aortocoronary conduits by CD68 + cells before their intraoperative application may be associated with higher risk of late graft occlusion. MMP2 might play a mediatory role in this process, with a greater increase seen in RA than ITA. Moreover, measuring of MMP location by immunohistochemistry is not sufficient to accurately estimate protein activity. Although gel zymography was proposed as a method of highly sensitive detection of gelatinases activity that enabled distinguishing between their active and zymogen forms, but getting reliable quantitative data with this technique was still challenging $[25,26]$.

\section{Acknowledgements}

This study was supported by State Committee for Scientific Research (Grant No. 5958/B/ /P01/2010/38). The funder had no role in study design, data collection and analysis, decision to publish, or preparation of the manuscript.

\section{Conflict of interest: None declared}

\section{References}

1. Huang F, Lai W, Chan C, et al. Comparison of bypass surgery and drug-eluting stenting in diabetic patients with left main and/or multivessel disease: A systematic review and metaanalysis of randomized and nonrandomized studies. Cardiol J. 
2015; 22(2): 123-134, doi: 10.5603/CJ.a2014.0036, indexed in Pubmed: 24846507.

2. Shavadia J, Norris CM, Graham MM, et al. Symptomatic graft failure and impact on clinical outcome after coronary artery bypass grafting surgery: Results from the Alberta Provincial Project for Outcome Assessment in Coronary Heart Disease registry. Am Heart J. 2015; 169(6): 833-840, doi: 10.1016/j. ahj.2015.02.022, indexed in Pubmed: 26027621.

3. Gaudino M, Benedetto U, Fremes S, et al. Radial-Artery or saphenous-vein grafts in coronary-artery bypass surgery. $\mathrm{N}$ Engl J Med. 2018; 378(22): 2069-2077, doi: 10.1056/nejmoa1716026.

4. Gansera B, Schmidtler F, Angelis I, et al. Patency of internal thoracic artery compared to vein grafts - postoperative angiographic findings in 1189 symptomatic patients in 12 years. Thorac Cardiovasc Surg. 2007; 55(7): 412-417, doi: 10.1055/s-2007-965372, indexed in Pubmed: 17902061.

5. Bonacchi M, Prifti E, Maiani M, et al. Perioperative and clinicalangiographic late outcome of total arterial myocardial revascularization according to different composite original graft techniques. Heart Vessels. 2006; 21(2): 69-77, doi: 10.1007/s00380-0050856-2, indexed in Pubmed: 16550306.

6. Cao C, Manganas C, Horton M, et al. Angiographic outcomes of radial artery versus saphenous vein in coronary artery bypass graft surgery: a meta-analysis of randomized controlled trials. J Thorac Cardiovasc Surg. 2013; 146(2): 255-261, doi: 10.1016/j. jtcvs.2012.07.014, indexed in Pubmed: 22871565.

7. Fonseca DA, Antunes PE, Cotrim MD. Ultrastructural and histomorphologic properties of the internal thoracic artery: implications for coronary revascularization. Coron Artery Dis. 2017; 28(6): 518-527, doi: 10.1097/MCA.0000000000000527, indexed in Pubmed: 28678142.

8. Heo $\mathrm{SH}$, Cho $\mathrm{CH}$, Kim HOk, et al. Plaque rupture is a determinant of vascular events in carotid artery atherosclerotic disease: involvement of matrix metalloproteinases 2 and 9. J Clin Neurol. 2011; 7(2): 69-76, doi: 10.3988/jcn.2011.7.2.69, indexed in Pubmed: 21779294.

9. Zhang L, Liao Mf, Tian L, et al. Overexpression of interleukin$1 \beta$ and interferon- $\gamma$ in type I thoracic aortic dissections and ascending thoracic aortic aneurysms: possible correlation with matrix metalloproteinase-9 expression and apoptosis of aortic media cells. Eur J Cardiothorac Surg. 2011; 40(1): 17-22, doi: 10.1016/j. ejcts.2010.09.019, indexed in Pubmed: 21349736.

10. Li Ya. Correlation analysis of levels of adiponectin and matrix metalloproteinase-9 with stability of coronary heart disease. Technol Health Care. 2015; 23 Suppl 1: S95-S98, doi: 10.3233/ thc-150937, indexed in Pubmed: 26410336.

11. Grzela T, Niderla-Bielinska J, Litwiniuk M, et al. The direct inhibition of MMP-2 and MMP-9 by an enzyme alginogel: a possible mechanism of healing support for venous leg ulcers. J Wound Care. 2014; 23(5): 278-285, doi: 10.12968/jowc.2014.23.5.278, indexed in Pubmed: 24810313.

12. Li TT, Xie Yi, Guo Y, et al. Effect of probucol on vascular remodeling due to atherosclerosis in rabbits: an intravascular ultrasound study. Chin Med J (Engl). 2011; 124(12): 1840-1847, indexed in Pubmed: 21740843.

13. Aoki T, Kataoka H, Morimoto M, et al. Macrophage-derived matrix metalloproteinase-2 and -9 promote the progression of cerebral aneurysms in rats. Stroke. 2007; 38(1): 162-169, doi: 10.1161/01.STR.0000252129.18605.c8, indexed in Pubmed: 17122420 .
14. Ghosh A, Pechota LV, Upchurch GR, et al. Cross-talk between macrophages, smooth muscle cells, and endothelial cells in response to cigarette smoke: the effects on MMP2 and 9. Mol Cell Biochem. 2015; 410(1-2): 75-84, doi: 10.1007/s11010-015-2539-3, indexed in Pubmed: 26318311.

15. Otsuka F, Kramer MCA, Woudstra P, et al. Natural progression of atherosclerosis from pathologic intimal thickening to late fibroatheroma in human coronary arteries: A pathology study. Atherosclerosis. 2015; 241(2): 772-782, doi: 10.1016/j.atherosclerosis.2015.05.011, indexed in Pubmed: 26058741.

16. Malinska A, Perek B, Buczkowski P, et al. CD68 expression in aortocoronary saphenous vein bypass grafts. Histochem Cell Biol. 2013; 140(2): 183-188, doi: 10.1007/s00418-012-1069-2, indexed in Pubmed: 23275124.

17. Perek B, Kowalska K, Kempisty B, et al. Gender and age-related variability of macrophage representation in the internal thoracic artery wall: does it matter? J Biol Regul Homeost Agents. 2018; 32(4): 791-802, indexed in Pubmed: 30043561.

18. Chung JiH, Jeon HJu, Hong SY, et al. Palmitate promotes the paracrine effects of macrophages on vascular smooth muscle cells: the role of bone morphogenetic proteins. PLoS One. 2012; 7(2): e29100, doi: 10.1371/journal.pone.0029100, indexed in Pubmed: 22363399.

19. Chatrou MLL, Cleutjens JP, van der Vusse GJ, et al. IntraSection analysis of human coronary arteries reveals a potential role for micro-calcifications in macrophage recruitment in the early stage of atherosclerosis. PLoS One. 2015; 10(11): e0142335, doi: 10.1371/journal.pone.0142335, indexed in Pubmed: 26555788.

20. Hinojosa-Amaya JM, Villarreal-Silva EE, Elizondo-Omana RE, et al. Conduits for myocardial revascularization grafts: The importance of morphology and imaging. Med Univ. 2010; 47(12): 115-119.

21. Ruengsakulrach P, Sinclair R, Komeda M, et al. Comparative histopathology of radial artery versus internal thoracic artery and risk factors for development of intimal hyperplasia and atherosclerosis. Circulation. 1999; 100(19 Suppl): II139-II144, doi: 10.1161/01. cir.100.suppl_2.ii-139, indexed in Pubmed: 10567293.

22. Perek B, Malinska A, Misterski M, et al. Preexisting high expression of matrix metalloproteinase-2 in tunica media of saphenous vein conduits is associated with unfavorable long-term outcomes after coronary artery bypass grafting. Biomed Res Int. 2013; 2013: 730721, doi: 10.1155/2013/730721, indexed in Pubmed: 24151618.

23. Turner NA, Hall KT, Ball SG, et al. Selective gene silencing of either MMP-2 or MMP-9 inhibits invasion of human saphenous vein smooth muscle cells. Atherosclerosis. 2007; 193(1): 36-43, doi: 10.1016/j.atherosclerosis.2006.08.017, indexed in Pubmed: 16979647.

24. Perek B, Malińska A, Ostalska-Nowicka D, et al. Cytokeratin 8 in venous grafts: a factor of unfavorable long-term prognosis in coronary artery bypass grafting patients. Cardiol J. 2013; 20(6): 583-591, doi: 10.5603/CJ.2013.0142, indexed in Pubmed: 24338534.

25. Toth M, Sohail A, Fridman R. Assessment of gelatinases (MMP2 and MMP-9) by gelatin zymography. Methods Mol Biol. 2012; 878: 121-135, doi: 10.1007/978-1-61779-854-2_8, indexed in Pubmed: 22674130.

26. Leber TM, Balkwill FR. Zymography: a single-step staining method for quantitation of proteolytic activity on substrate gels. Anal Biochem. 1997; 249(1): 24-28, doi: 10.1006/abio.1997.2170, indexed in Pubmed: 9193704. 